\title{
Improving Touchscreen Keyboards: Design issues and a comparison with other devices
}

\author{
Andrew Sears \\ Human-Computer Interaction Laboratory \\ Department of Computer Science \\ University of Maryland \\ College Park, MD 20742
}

March 11, 1991

\begin{abstract}
This study explored touchscreen keyboards using high precision touchscreen strategies. Phase one evaluated three possible monitor positions: 30, 45, and 75 degrees from horizontal. Results indicate that the 75 degree angle, approximately the standard monitor position, resulted in more fatigue and lower preference ratings. Phase two collected touch bias and key size data for the 30 degree angle. Subjects consistently touched below targets, and touched to the left of targets on either side of the screen. Using these data, a touchscreen keyboard was designed. Phase three compared this keyboard with a mouse activated keyboard, and the standard QWERTY keyboard for typing relatively short strings of 6, 19, and 44 characters. Results indicate that users can type approximately 25 words per minute with the touchscreen keyboard, compared to 17 WPM using the mouse, and 58 WPM when using the keyboard. Possible improvements to touchscreen keyboards are suggested.
\end{abstract}




\section{Introduction}

\section{Overview}

Many studies have compared input devices. Touchscreens have been compared to mice, lightpens, keyboards, and joysticks (Sears \& Shneiderman, 1991; Battenberg, 1989; Beringer, 1989; Ostroff \& Shneiderman, 1988; Ahlström \& Lenman, 1987; Muratore, 1987; Karat, McDonald, \& Anderson, 1986). Many studies compare devices for simple tasks, such as cursor positioning. Recent work by Sears and Shneiderman (1991) investigated the use of touchscreens for tasks requiring accurate selection of small targets. The study compared high precision touchscreens to a mouse for selecting various size targets. Results indicate that touchscreens are as fast and accurate as a mouse when selecting targets as small as $1.7 \times 2.2 \mathrm{~mm}$ and that touchscreens can be used for many tasks that currently are performed with a mouse. While studies of this nature are useful, experiments investigating more realistic tasks may provide more valuable information for application designers.

Several studies have investigated the use of touchscreens for data entry (Weisner, 1988; Gould, Greene, Boies, Meluson, \& Rasamny, 1990). Comparisons are typically between a touchscreen keyboard and a standard QWERTY keyboard. While touchscreens have been slower than standard keyboards for typing, there are situations where using a touchscreen for data entry may be useful. For example, applications requiring infrequent data entry. For these applications the keyboard may be used infrequently while consuming a large amount of work space. If a touchscreen were used, a keyboard could be presented only when needed, and other interfaces could be used when appropriate, allowing more flexible interaction with the computer. Touchscreens may also be useful when keyboards may not be practical. Keyboards are not durable enough to be used in many public access systems while touchscreens have been used successfully in public access systems for years. Portable computers, which can not use mice effectively, may also benefit from using touchscreens. Touchscreen keyboards also allow the keyboard to be customized to the users' preference, QWERTY, Dvorak, French, Swedish, or any other keyboard the user wants.

This study investigates the use of touchscreen keyboards for limited data entry. Several design issues were addressed to develop an improved touchscreen keyboard. The mounting angle of the monitor, size of keys, and the location of touchable regions relative to visible targets were all explored. Using data collected, a touchscreen keyboard was designed and compared to a standard keyboard and a mouse activated keyboard. Error rates and typing speeds were collected. This work will provide valuable information about the angle at which users prefer to work with touchscreens, minimum key sizes for low error rates when using the lift-off selection strategy, and a first estimate of the typing rates achievable with touchscreen keyboards.

\section{$\underline{\text { Relevant studies }}$}

Mounting Angle

The first issue of interest was the mounting angle for the touchscreen. A study by Ahlström and 
Lenman (1990) investigated the effect screen angle has on user performance and fatigue. Subjects repeatedly performed simple menu selection tasks with the touchscreen mounted at 90, 60, 45, 30, 22.5, and 0 degrees from horizontal. Results indicate that mounting the touchscreen at 30 degrees results in less fatigue than any of the other angles. Positioning times show no large differences between the angles, while error data indicates that 0 and 90 degree angles result in more errors. This study is still in the early stages, but the data collected so far indicate that 30 degrees may prove optimal.

Tinker (1956) compared reading speeds from paper at 45, 30, -10, and -30 degrees from horizontal. Results indicate that reading was fastest at a 45 degree angle, and that visibility of the characters was highest in this position (using the Luckiesh-Moss Visibility Meter). Grant (1987) has suggested that screen placements lower than the standard monitor position may also reduce eye strain.

The fact that the standard monitor position is not optimal, at least when using a touchscreen, is supported by these studies. The first phase of the current experiment compares three monitor positions chosen based on previous research, 30, 45, and 75 degrees from horizontal ( 75 is approximately the standard monitor position), for perceived fatigue and user preference. The hypothesis is that the 30 degree angle will result in less fatigue and be preferred by users.

\section{Biases}

Once the monitor position has been determined, biases that may be introduced by this position, and key sizes must determined. Biases are consistent differences between the location users want to touch, and where they actually touch (Figure 2). Previous studies indicate that touch biases exist for both the $\mathrm{X}$ and $\mathrm{Y}$ axes, varying with the position of users relative to the monitor. Beringer and his colleagues (with Peterson in 1985, and Bowman in 1989) investigated the effect screen angle has on touch biases in the $\mathrm{Y}$ axis. Mounting the monitor perpendicular to the line of sight resulted in virtually no bias in the $\mathrm{Y}$ direction. As the monitor is tilted away from users, they begin touching below the target. As the monitor is tilted towards users, they touch above the target. Beringer and Bowman (1989) recommend three possible solutions for this bias: limiting the resolution of the tasks to be performed, compensating for predicted bias using software, or operator training. A combination of limiting the resolution of tasks, and compensating for biases in software should prove effective.

Hall, Cunningham, Roache, and Cox (1988) also studied biases created by users at various positions relative to the touchscreen. Results indicate that touch biases in both the $\mathrm{X}$ and $\mathrm{Y}$ axes depend on viewing angle, but the effect target location has on biases was not investigated. Sitting to the left, right, or in the center of the monitor resulted in different biases on the $\mathrm{X}$ axis, while viewing from different vertical angles resulted in different biases on the $\mathrm{Y}$ axis. This work, and that by Beringer et al. (1985, 1989), supports the need to investigate touch biases. Bias data will be collected for the monitor position selected after the first phase, and used when designing the touchscreen keyboard. It is expected that biases will exist for both the $\mathrm{X}$ and $\mathrm{Y}$ axes, and that these biases will vary depending on the location of the target on the screen. 
Key Sizes

Determining a key size that can easily be touched will allow the design of effective interfaces, with low error rates. Hall et al. (1988) collected data about accuracy for various user positions and concluded that targets $26 \mathrm{~mm}$ per side result in over $99 \%$ accuracy when users are sitting in front of the monitor. Gould, Greene, Boise, Meluson, and Rasamny (1990) investigated the use of touchscreen keyboards using a minimum key size of 20x25 mm. These studies indicate that keys should be at least $20 \mathrm{~mm}$ per side to minimize errors. However, none of these studies explicitly corrected for touch biases created by the monitor position. This study will investigate the interaction between correcting for biases and minimum key size. It is believed that by correcting consistent biases, the size of a key can be reduced without increasing the error rate.

\section{Touchscreen Keyboards}

Gould et al. (1990) investigated using touchscreens for simple tasks, including typing on a touchscreen keyboard. Comparisons were made, using data from previous studies, between performance of touchscreen keyboards and standard keyboards. Comparing typing times for a touchscreen keyboard (1.0 seconds/key, 12 WPM) to times for a standard keyboard (0.6 s/key, $20 \mathrm{WPM}$ ), they found that a touchscreen keyboard requires $67 \%$ longer. The low typing speed on the keyboard, $20 \mathrm{WPM}$, indicates that the tasks used may have involved more effort than simply entering data or that users were simply not familiar with keyboards. Additional studies have compared the touchscreen and keyboard, but little work has been done to measure typing rates.

This study will investigate using touchscreen keyboards for limited data entry. Although it is unlikely that touchscreens will be useful for extensive data entry, there are situations where a touchscreen keyboard may prove useful. The purpose of measuring typing rates is to provide an initial estimate of the typing rate users can easily attain using a touchscreen keyboard.

\section{Experiment}

\section{Introduction}

This experiment was divided into three phases. During Phase one, subjects used a touchscreen mounted at three angles: 30, 45, and 75 degrees from horizontal. The purpose of Phase one was to identify a monitor position users felt was less fatiguing, and preferred. Phase two identified touch biases when the monitor is at a 30 degree angle, the angle selected in Phase one. Phase two also allowed a key size that would result in reduced errors to be determined. A touchscreen keyboard was designed, using the key size determined, and compensating for biases identified. Phase three compared typing speeds and error rates for the touchscreen keyboard, a standard keyboard, and a mouse activated keyboard.

The purpose of this experiment was to demonstrate that screen angle and touch biases are important considerations when designing touchscreen applications, and to make a first attempt at an improved touchscreen keyboard. It is expected that the 75 degree angle will prove more fatiguing, and 
users will prefer the 30 degree angle. In addition, an estimate of typing speeds for the touchscreen will be provided. The standard keyboard is expected to be fastest, and the mouse slowest. Possible improvements to touchscreen keyboards will be identified.

\section{Equipment}

A NEC PowerMate 386/25 PC with a Sony Multi-scan HG monitor and MicroTouch touchscreen was used for this experiment. A special desk that allowed the monitor to be mounted below the surface of the desk was used (Figure 1). The keyboard fit in a drawer that slid into the desk when not in use, and was in a normal typing position when needed. The monitor measured $27.2 \times 19.8 \mathrm{~cm}$ and was used in VGA mode (640x480 pixels). The distances on the $\mathrm{X}$ and $\mathrm{Y}$ axes were measured from the upper left corner. The MicroTouch touchscreen is a capacitive touchscreen that provides continuous information about the location of a touch on a 1024x1024 grid. It requires only a light touch to be activated and averages the location of all simultaneous touches, returning a centroid location. The touchscreen was cleaned once before the first subject began each phase and was not cleaned at any other time during the experiment. Software was written to convert the touchscreen coordinates to pixel coordinates, and to correct for biases determined in Phase two. A standard mechanical mouse with two buttons was used. The mouse was calibrated so a $15 \mathrm{~cm}$ horizontal movement moved the cursor across the screen horizontally, and a $11 \mathrm{~cm}$ vertical movement moved the cursor across the screen vertically.

\section{Phase One - Determining the screen angle Introduction}

The purpose of Phase one was to determine the mounting angle for the touchscreen keyboard. User preferences and fatigue rankings were collected. Three angles were tested: 30, 45, and 75 degrees from horizontal. The 30 and 45 degree angles were chosen based on previous experience with touchscreens, and current research by Ahlström and Lenman (1990). The 75 degree angle was chosen to approximate the angle at which many screens are normally mounted.

\section{Subjects}

Due to the nature of Phase one, previous experience with computers or touchscreens was not important. Ten computer science students and staff members at the University of Maryland volunteered to participate in Phase one. Six subjects were familiar with touchscreens.

\section{Design and Procedure}

When mounted at 30 or 45 degrees, the monitor was partially below the surface of the desk and many subjects rested their forearms on a bar in front of the monitor, possibly reducing fatigue (Figure 1). When mounted at 75 degrees, the monitor was on top of the desk and several subjects rested their elbows on the table in front of the monitor. 


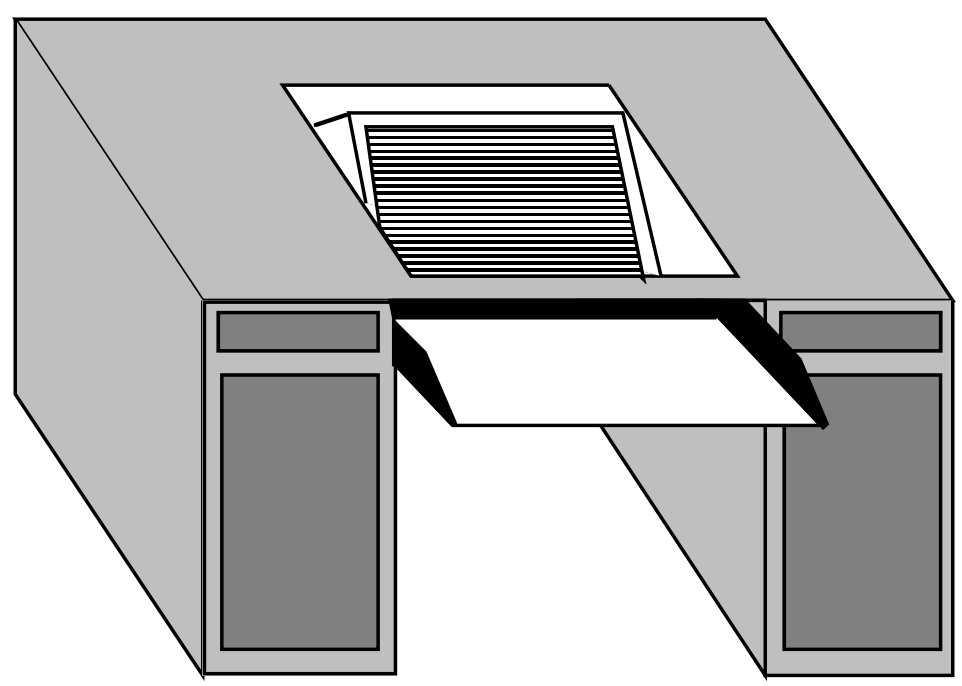

Figure 1 - Desk used for experiment

Each subject used all three screen angles, in random order, with an adequate delay between angles to prevent a sequence effect. Every subject was required to touch seventy small targets (1.65 mm square) presented in a 10 by 7 matrix ( 10 horizontal locations, 7 vertical) twice. Although it is very difficult to accurately touch targets of this size, the purpose was not to touch the targets accurately, but simply to use the touchscreen for an extended task. The order that the one hundred forty targets were presented was randomized to prevent biases. Subjects were instructed to touch each target without stressing time or accuracy. After users touched the screen and lifted their fingers, a sound was generated and the next target was presented.

When subjects completed Phase one they were asked to rank the three screen angles for fatigue ( $1=$ least fatiguing, $3=$ =most fatiguing) and preference for extended use ( $1=$ first preference, $3=$ third preference).

\section{Results}

\section{Preference and Fatigue Rankings}

Preference and fatigue rankings are presented in Tables 1 and 2 respectively. Angles were ranked from one to three ( $1=$ preferred angle, $1=$ least fatiguing angle). Freidman's two way analysis of variance was performed on both rankings. Significant effects of screen angle for both fatigue $F(2)=13.4$ and preference $\mathrm{F}(2)=10.4$ were found $(\mathrm{p}<.01)$. The Wilcoxon signed ranks test was performed to determine any significant differences. When mounted at the 75 degree angle, the touchscreen received the lowest preference ranking and was considered the most fatiguing $(\mathrm{p}<.05)$. No other significant differences were found. 


$\begin{array}{cccccc}\text { Angle } & \text { Mean } & \text { Standard Deviation } & \text { Angle } & \text { Mean } & \text { Standard Deviation } \\ 30 & 1.4 & 0.52 & 30 & 1.3 & 0.48 \\ 45 & 1.8 & 0.79 & 45 & 1.8 & 0.63 \\ 75 & 2.8 & 0.42 & 75 & 2.9 & 0.32 \\ \text { Table } 1 \text { - Preference Rankings } & \text { Table } 2 & \text { - Fatigue Rankings } \\ & 1 \text { - preferred } & & 1=\text { least fatiguing }\end{array}$

\section{Discussion}

Results indicate that 30 and 45 degree angles result in lower perceived fatigue, and higher user preference rankings. The 30 degree angle was chosen for the touchscreen keyboard based on the distribution of rankings for preference and fatigue. The 30 degree angle was ranked 6-4-0 for preference ( 6 ranked it best, 0 worst) while the 45 degree angle was ranked 4-4-2 (4 best, 2 worst). Fatigue rankings similarly favored the 30 degree angle. The 30 degree angle was ranked 7-3-0 ( 7 felt it caused the least fatigue, 0 felt it caused the most), and the 45 degree angle was ranked 3-6-1 (3 least, 1 most).

As expected, the 75 degree angle was rated as the most fatiguing and least preferred. Although the 30 degree angle was not significantly better than the 45 degree angle, it did received better ratings on average. The most important result from this phase is the additional confirmation that the standard monitor position is sub-optimal, at least when used with touchscreens.

\section{Phase Two: Determining touch biases and key size Introduction}

Phase two was conducted to collect touch bias and key size data for a touchscreen mounted at 30 degrees from horizontal (the angle chosen in Phase one). Range data will allow us to determine the distribution of where users' actually touched compared to where they were trying to touch (Figure 2). This distribution will allow us to determine how large a key must be to capture a predetermined percentage of the touches.

Touch bias data is intended to indicate if subjects consistently miss the desired target in one direction (Figure 2). Mounting the touchscreen at an angle other than perpendicular to the subjects' line of sight may introduce a small bias due to parallax (Figure 2). Researchers have found not only vertical biases, but horizontal biases also (Hall, Cunningham, Roache, \& Cox, 1988; Beringer \& Peterson, 1985; Beringer \& Bowman, 1989). By correcting consistent biases, it should be possible to reduce the size of the keys without increasing error rates. 


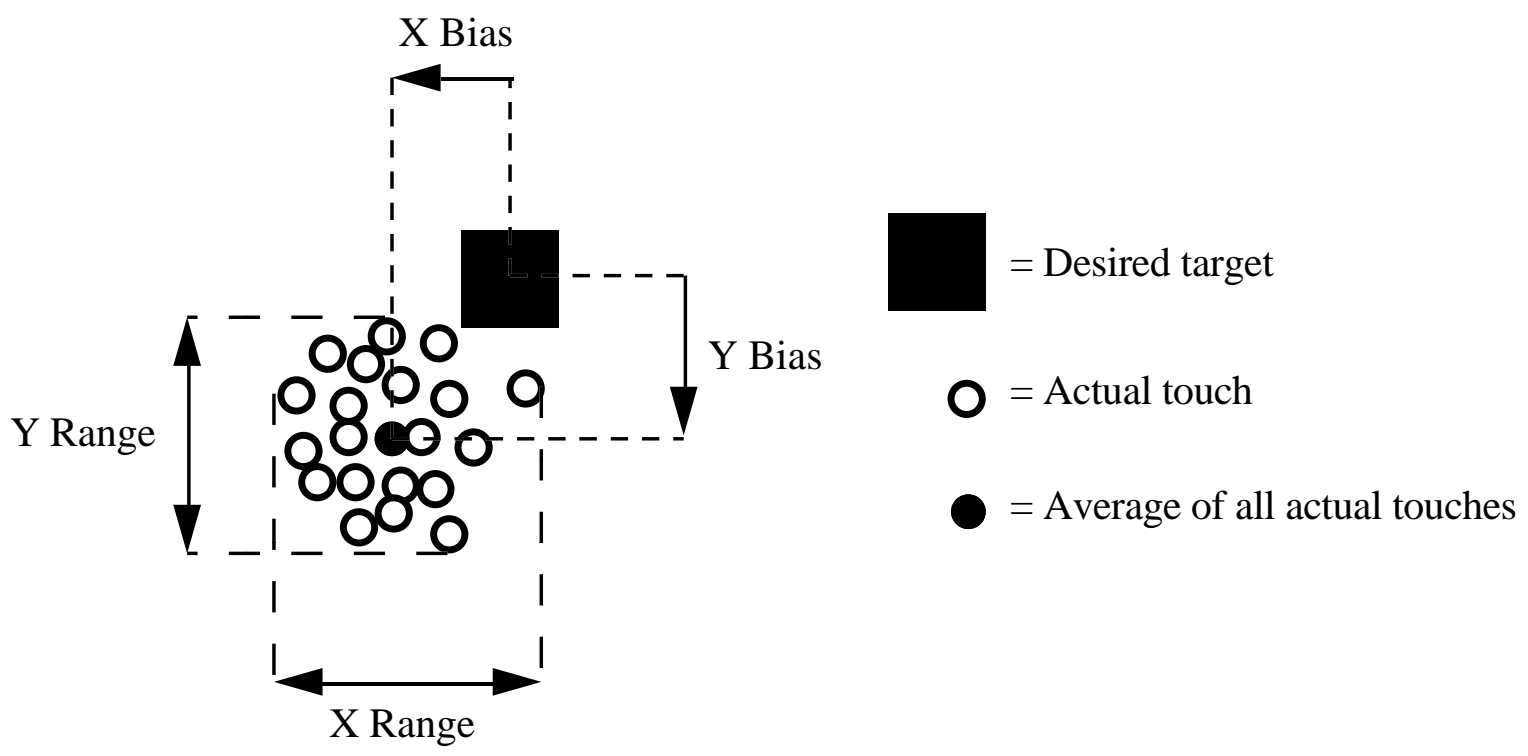

Figure 2 - Diagram of what biases and ranges represent.

\section{Subjects}

Due to the nature of Phase two, previous experience with touchscreens, or computers in general was not important. Fourteen students and staff members of the Computer Science Department at the University of Maryland volunteered to participate as subjects. Eight subjects were familiar with touchscreens, three used them on a regular basis. Ten subjects were right handed, four left handed. Eight subjects were informally questioned about eye dominance after the experiment in an attempt to determine if eye dominance explained the results.

\section{Design and Procedure}

The screen was mounted at a 30 degree angle, allowing subjects to rest their arms on a bar in front of the monitor (Figure 1). Every subject was required to touch seventy small targets (1.65 mm square) presented in a 10 by 7 matrix (10 horizontal locations, 7 vertical) twice. Although it is very difficult to accurately touch targets of this size, using these small targets allow more precise calculations of the distance between where users were trying to touch and where they actually touched. If larger targets were used, it would be difficult to know if subjects actually tried to touch the center of the target, or possibly a corner. The order that the one hundred forty targets were presented was randomized to prevent biases. For this phase, subjects were instructed to touch the screen in a natural way, but to try to be accurate. After users touched the screen and lifted their fingers, a sound was generated and the next target was presented. The distance from the location of the first touch, to the center of the target was recorded automatically.

\section{Results}

$\underline{\text { Touch Biases }}$ 
Touch bias means appear in Tables 3 and 4. Touch bias means represent the distance from the desired touch location (center of the target) to the actual touch location (Figure 2). A positive touch bias on the $\mathrm{X}$ axis indicates that users touched to the right of the target. A positive bias for the $\mathrm{Y}$ axis indicates that users touched below the target. X position represents how far from the left of the screen subjects were touching. Y position represents how far from the top of the screen subjects were touching.

$\begin{array}{cl}\text { X Position }(\mathrm{cm}) & \text { Mean Bias }(\mathrm{cm}) \\ 0.83 & -0.41 \\ 3.55 & -0.33 \\ 6.27 & -0.17 \\ 8.99 & -0.08 \\ 11.72 & 0.00 \\ 14.44 & -0.04 \\ 17.16 & -0.17 \\ 19.88 & -0.21 \\ 22.61 & -0.33 \\ 25.33 & -0.45\end{array}$

Table 3 - Touch Biases for X axis for a 30 degree monitor angle (in $\mathrm{cm}$ ). Negative bias means touch was to the left of the target.

$\begin{array}{cl}\text { Y Position }(\mathrm{cm}) & \text { Mean Bias }(\mathrm{cm}) \\ 1.24 & +0.54 \\ 4.13 & +0.54 \\ 7.01 & +0.49 \\ 9.90 & +0.45 \\ 12.79 & +0.41 \\ 15.68 & +0.41 \\ 18.56 & +0.41\end{array}$

Table 4 - Touch Biases for Y axis for a 30 degree monitor angle (in $\mathrm{cm}$ ). Positive bias means touch was below target.

\section{$\underline{\text { Ranges }}$}

Range data appears in Tables 5 and 6. X position represents how far from the left of the screen subjects were touching. Y position represents how far from the top of the screen subjects were touching. Ranges represent the extreme misses on either side of a target (the $\mathrm{X}$ range indicates the farthest that anyone missed to the right of the target minus the farthest they missed to the left, see Figure 2).

$\begin{array}{cl}\text { X Position }(\mathrm{cm}) & \text { Range }(\mathrm{cm}) \\ 0.83 & 1.28 \\ 3.55 & 2.48 \\ 6.27 & 1.32 \\ 8.99 & 1.44 \\ 11.72 & 1.36 \\ 14.44 & 1.40 \\ 17.16 & 1.77\end{array}$




$\begin{array}{ll}19.88 & 1.24 \\ 22.61 & 2.15 \\ 25.33 & 1.57\end{array}$

Table 5 - Ranges for $\mathrm{X}$ axis for a 30 degree monitor angle (in $\mathrm{cm}$ ). Maximum range subjects missed targets by for various $\mathrm{X}$ positions.

$\begin{array}{cl}\text { Y Position }(\mathrm{cm}) & \text { Range }(\mathrm{cm}) \\ 1.24 & 1.90 \\ 4.13 & 1.53 \\ 7.01 & 1.40 \\ 9.90 & 1.90 \\ 12.79 & 1.49 \\ 15.68 & 1.90 \\ 18.56 & 1.49\end{array}$

Table 6 - Ranges for Y axis for a 30 degree monitor angle (in $\mathrm{cm}$ ). Maximum range subjects missed targets by for various $Y$ positions.

\section{Discussion}

Using the biases and keys of an appropriate size should allow accurate key touches. The biases measured in this phase indicate that users consistently touch below the desired target. Users also touch to the left of targets on either side of the screen. There are many possible explanations for these biases. Parallax, although greatly reduced compared to earlier touchscreens, may explain the vertical biases (Y axis). When the monitor is mounted at 30 degrees from horizontal, the users' line of sight was approximately 38 degrees from orthogonal with the monitor surface. The extra hardware mounted on the touchscreen used in this study is approximately $0.64 \mathrm{~cm}$ thick, accounting for bias of approximately +0.49 $\mathrm{cm}$ (below the target). This is very close to the vertical biases measured in this phase which varied from +0.41 to $+0.54 \mathrm{~cm}$. The horizontal biases ( $\mathrm{X}$ axis) are more difficult to explain. Left vs. right handedness and eye dominance do not appear to explain these biases. The results obtained in this study differ from those of Hall et al. (1988) and Beringer et al. $(1985,1989)$ possibly due to the different touchscreen technologies used. Although we are unable to explain the horizontal biases, they were consistent for all users in this study, and will be used to correct touch locations. Although both vertical and horizontal biases will vary depending on touchscreen technology, manufacturer, position relative to user, and application, it is important to realize that biases exist, and may need to be corrected if fast

accurate selections are necessary. Biases will be used to adjust the location of actual touches to be closer to where users' are trying to touch.

By using the location of all actual touches, we calculated square keys measuring $2.61 \mathrm{~cm}$ per side were required to capture all touches (Figure 3). These results are similar to those of Hall et al., who report that targets must be $2.6 \mathrm{~cm}$ square to result in over $99 \%$ accuracy. However, we believed that correcting for biases would allow the size of the key to be reduced without increasing the error rate. Part of the motivation for this was the range data collected. For instance, the largest range measured was $2.48 \mathrm{~cm}$, indicating that a square $2.48 \mathrm{~cm}$ per side would capture every touch if it were placed strategically for every 
key on the screen (Figure 3). By shifting the touchable regions for all target by the appropriate biases, depending on target location, keys on a QWERTY keyboard were be reduced to $2.27 \mathrm{~cm}$ per side while maintaining an error rate of less than $1 \%$ (this does not count spelling errors, it only counts errors created when users try to touch a key and accidentally touch another) (Figure 3). Therefore, the touchable regions for the keys used in Phase three were offset using the bias data collected in this phase. Typically the touchable regions were shifted slightly lower on the screen than the actual key, and were shifted slightly to the left of the keys on either side of the screen.

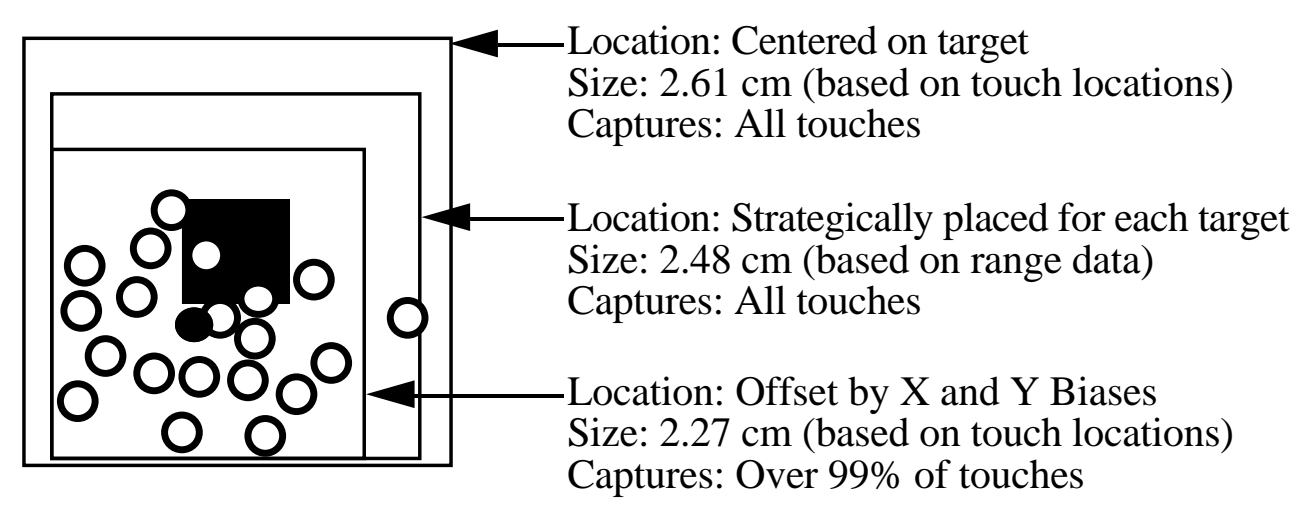

Figure 3 - Diagram of possible square key sizes

\section{Phase Three - Data Entry Comparison}

\section{Introduction}

The purpose of Phase three was to measure data entry rates for three input devices, a touchscreen, a mouse, and a standard QWERTY keyboard. When using the touchscreen and mouse, a QWERTY keyboard was presented on the screen and data was entered by selecting keys on that keyboard (Figure 4). 


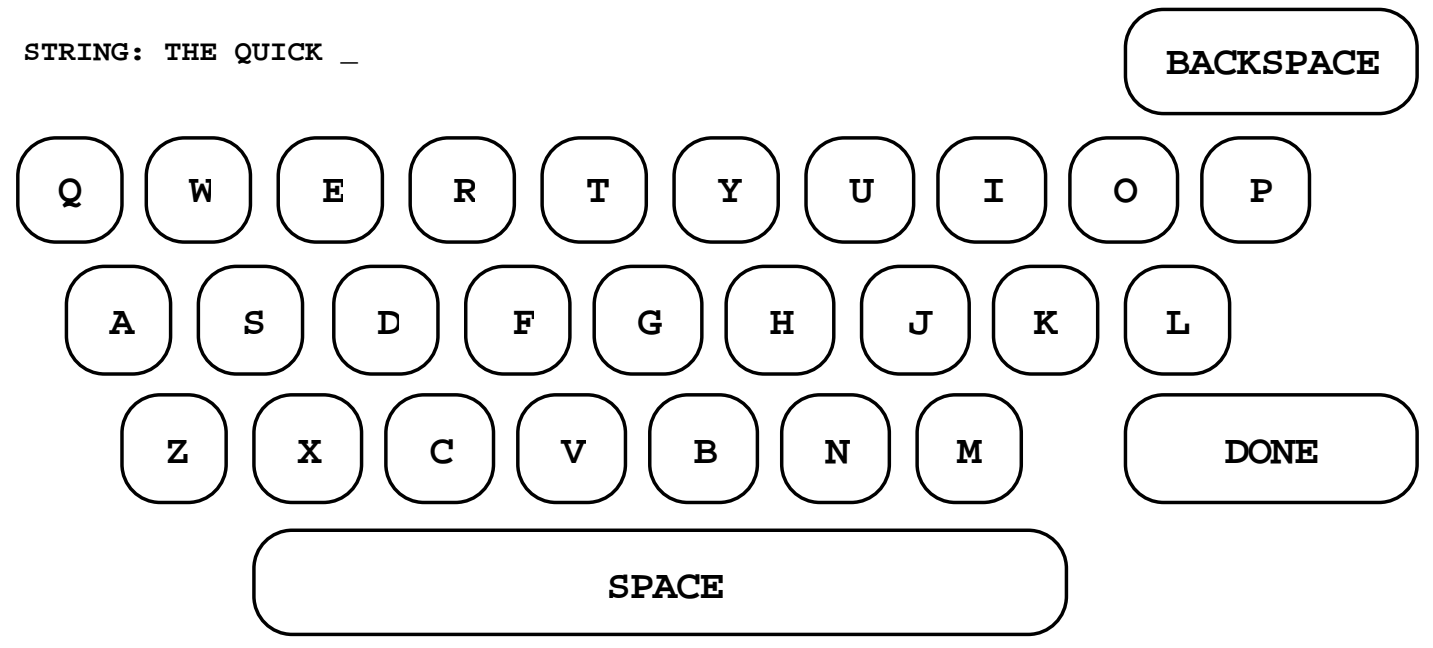

Figure 4 - QWERTY keyboard as it appeared on the screen with the string to be entered, and the beginning of the string as it was entered by the subject

\section{Subjects}

Nine graduate students, staff, and faculty members of the Computer Science Department at the University of Maryland volunteered to participate as subjects. The purpose of this study was to compare how frequent users perform with each input device. For this reason, novice computer users were not recruited. All subjects were familiar with the QWERTY keyboard and mouse, and had used touchscreens previously. However, few of the subjects used a touchscreen on a daily basis or had used a touchscreen keyboard previously.

\section{Design and Procedure}

For Phase three, the monitor was mounted at 30 degrees from horizontal. Alphabetic keys were $2.27 \mathrm{~cm}$ square, and the touchable region for each key was offset using the touch biases from Phase two. The space bar, backspace and done keys were all proportional in size to the alphabetic keys. The touchscreen keyboard was designed to use the land-on selection strategy, resulting in selections at the point where users' fingers first touch the screen, if they touch a key. Both visual and audible feedback were provided to aid users. When a key was touched, it flashed (inverted briefly) and a short tone was made. This allowed users to get confirmation that a key was touched audibly, or that the correct key was touched visually.

Input device and strings entered were within subject variables. Each subject entered one practice string with each input device, and then used each device to enter six strings (Table 7). Input device and string order were randomized to prevent possible biases. All subjects participated in the experiment three to five times, spaced over four or five days. This allowed data to be collected for the first exposure to each interface, as well as data entry rates after limited exposure. Subjects were instructed to be fast, but accurate, as they would when performing a real task. 
The time from when the first character was entered until the done (return) key was entered was recorded for every string and input device combination. The number of corrected and uncorrected errors were recorded for each string and device combination. A sequence of consecutive backspaces was considered one corrected error. Any error in the final string entered was considered an uncorrected error.

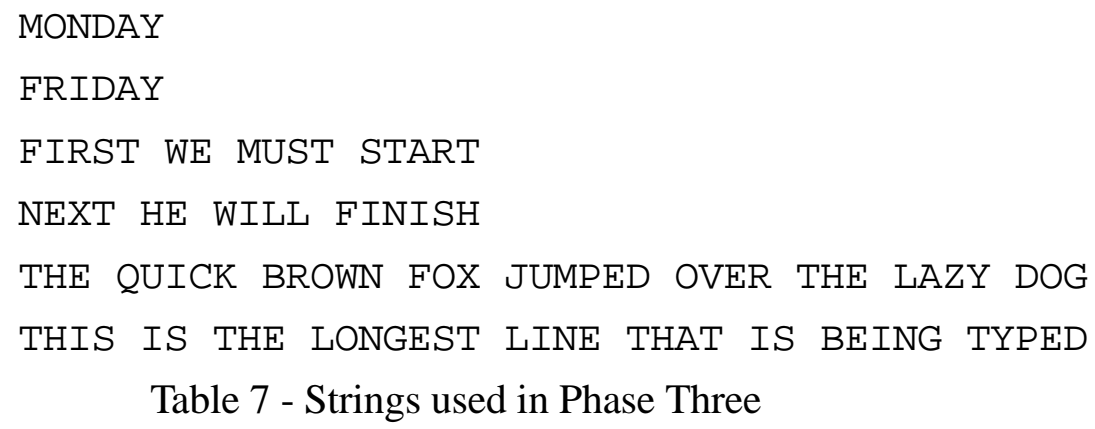

\section{Results}

\section{$\underline{\text { Selection Times }}$}

Mean typing times and standard deviations for the first and last trial appear in Table 8. An ANOVA with repeated measures for input device showed a significant main effect for input device for both the first and last trials $[\mathrm{p}<0.005, \mathrm{~F}(2,16)=138.904 ; \mathrm{p}<0.005, \mathrm{~F}(2,16)=338.992$ respectively]. Tukey's post hoc HSD test showed that the keyboard was faster than both the touchscreen and mouse, and that the touchscreen was faster than the mouse for both trials $(\mathrm{p}<0.005)$.

\begin{tabular}{|c|c|c|c|c|}
\hline \multirow{3}{*}{$\begin{array}{l}\text { Trial } \\
\text { First }\end{array}$} & \multicolumn{2}{|c|}{ Input Device } & \multirow[b]{2}{*}{ Mouse } & \\
\hline & Touchscreen & Keyboard & & \\
\hline & $68.07 \quad(10.61)$ & $32.06 \quad(12.48)$ & 105.15 & $(21.06)$ \\
\hline Last & $65.24 \quad(9.13)$ & $28.45 \quad(11.07)$ & 96.76 & $(8.94)$ \\
\hline
\end{tabular}

\section{Error Rates}

Errors were divided into two categories, corrected and uncorrected. Errors from all six strings were combined for each input device. An ANOVA was performed on uncorrected, corrected, and total errors. No significant differences were found. The mean number of total errors (for all six strings), for the first and last trial combined, was 0.9 for the mouse, 1.4 for the keyboard, and 1.8 for the touchscreen.

\section{Discussion}

Many subjects began by trying to "touch-type" on the touchscreen as they would on a standard keyboard. They soon realized that this was not practical. By the time the practice trials were completed, all subjects were using both hands and between two and eight fingers to type on the touchscreen. The 
majority of the subjects used four or six fingers when typing on the touchscreen.

Typing times were converted to words per minute (WPM), assuming 5 characters per word. Mean typing speeds (WPM) for the last trial are 17.1, 25.4, and 58.2 WPM for the mouse, touchscreen, and keyboard respectively (Figure 5). These typing rates compare favorably with those reported by Gould et al. (1990), who reported typing speeds equivalent to 12 WPM for the touchscreen and 20 WPM for the keyboard. These differences are probably due, at least in part, to the different users, keyboard layout, and tasks performed.

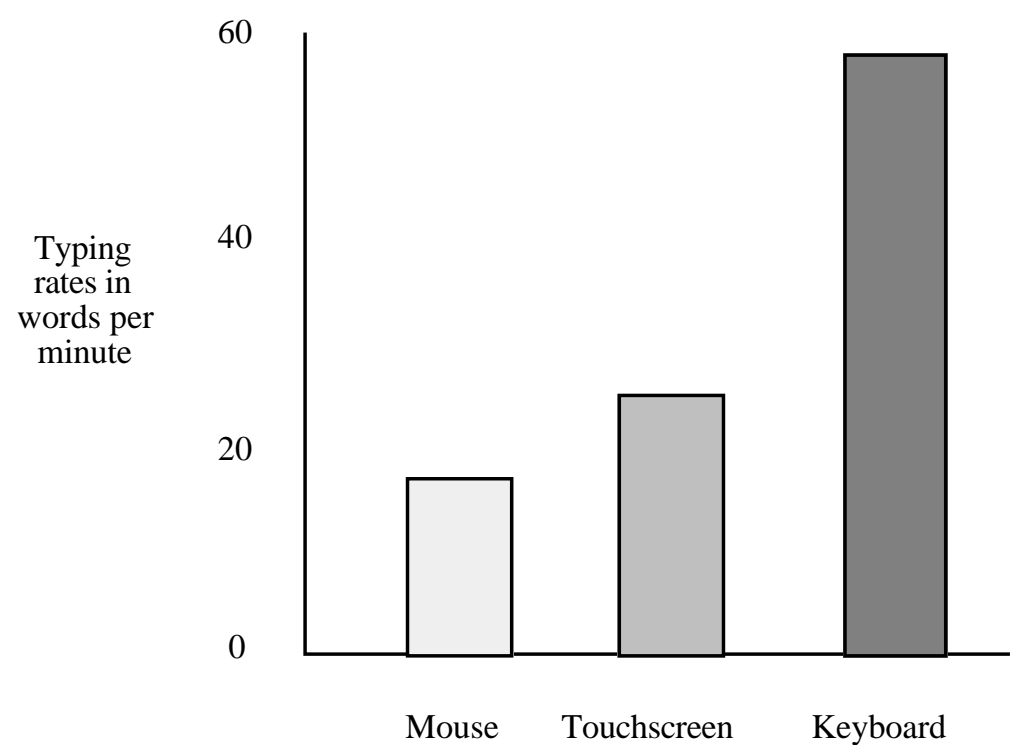

Figure 5 - Typing rates in words per minute for three input devices

Comparing the time per key to previous studies of selection times for the touchscreen (Sears \& Shneiderman, 1991; Muratore, 1987) is also encouraging. Converting the results from the current study to $\mathrm{s} /$ key results in $0.47 \mathrm{~s} / \mathrm{key}$ for the touchscreen. This is considerably faster than what would be predicted from previous studies. This discrepancy may also be explained by the different tasks performed. In many selection time studies, users touched randomly placed targets, and would not know where a target would appear ahead of time. This forced users to visually scan the screen to locate each target. When using a touchscreen keyboard, users know the next letter they wish to type, and where it is located. This allows users to immediately start moving to the next key, reducing selection times considerably.

The significant difference in the typing rates for the touchscreen and mouse may be caused by a fundamental difference between the devices, their directness. Touchscreens allow users to make selections by pointing directly at the object of interest. There is no need to convert their movements to movements of the cursor on the screen, allowing more natural selections. In addition, when using a mouse, users must drag a cursor from one location to another. However, when using a touchscreen, a cursor is present 
only when users' fingers are on the screen, if ever. When users lift their fingers after making a selection, the cursor disappears, and does not reappear until they touch the screen again. This allows users to move their fingers directly to the next character to be entered, without dragging anything across the screen. The freedom from dragging a cursor allows very fast, natural movements.

Although at first it may appear that a smaller keyboard would result in faster typing times when using the mouse, this is not necessarily true. Fitts' Law predicts selection time as a function of the distance (D) to the target, and the width (W) of the target, $T=a+b[\log (2 D / W)]$ and has been shown to be accurate when using a mouse (Fitts', 1954; Card, Moran, \& Newell, 1983). If the size of the keyboard were reduced by $50 \%$ in both the $\mathrm{X}$ and $\mathrm{Y}$ dimensions, the distance to a target is halved, but the size of the target is also halved, leaving the selection time the same. Therefore, reducing the size of the keyboard should not have a significant effect on typing speeds when using a mouse. However, reducing the size of the keyboard may result in slower typing times when using a touchscreen. A proposed change to Fitts' Law for touchscreens, $\mathrm{T}=\mathrm{a}+\mathrm{b}[\log \mathrm{D} / \mathrm{W}]+\mathrm{d}[\log (\mathrm{e} / \mathrm{W})]$, suggests that there are two components to a pointing action with a touchscreen (Sears \& Shneiderman, 1991). The first measures the fast movement of the hand to the screen, the second measures the slower action of making small adjustments to the location being selected. With a large keyboard, like the one used in this study, there is no time needed to adjust the location once the screen has been touched. If the keyboard is small, and the lift-off strategy is used to allow more precise touches, then the time to adjust the location of the touch will increase. This could result in slower overall typing speeds for small touchscreen keyboards. A study is under way that will investigate the size of the touchscreen keyboard on typing times (Revis, Swatski, Crittenden, Sears, \& Shneiderman, 1991).

\section{Conclusions}

Phase one confirmed that the standard monitor position is sub-optimal, at least when using a touchscreen. It is suggested that an angle of 30 degrees from horizontal may result in the least fatigue and highest preference ratings. However, additional angles must be studied before the optimal angle can be determined.

Phase two demonstrated that although touch biases may be small, and will change depending on the touchscreen hardware, manufacturer, application, and position of the user, it is important to understand that biases exist and may impact user performance. It is possible in many situations to get a rough measurement of the biases that exist and correct for them. In this study correcting for biases allowed keys to be reduced from $2.61 \mathrm{~cm}$ per side to $2.27 \mathrm{~cm}$ per side while maintaining an error rate of less than $1 \%$. Similar benefits are possible in other applications, especially when selections are made when users first touch the screen.

The final phase of this experiment demonstrated the potential of touchscreen keyboards. Although not as fast as standard keyboards, touchscreen keyboards may be useful in certain situations. When limited text entry is needed, or standard keyboards may be awkward, a touchscreen keyboard may be a 
viable alternative. It has been demonstrated that users can type as many as 25 words per minute on the keyboard designed in this study. A more recent investigation into the effect of keyboard size on typing speeds indicates that users can actually type as many as 30 WPM with limited experience on the same size keyboard and as fast as $20 \mathrm{WPM}$ on a keyboard that is only $7 \mathrm{~cm}$ wide (Revis, Swatski, Crittenden, Sears, \& Shneiderman, 1991).

\section{Impact on practitioners and researchers}

This study demonstrated the potential for touchscreen keyboards. It also provided additional data supporting the claims that touchscreens should not be mounted in the standard monitor position, and that correcting for biases will allow designers to use smaller buttons without increasing error rates. Touchscreens are becoming more attractive because of the availability of high precision strategies that allow more and smaller targets per screen, while increasing accuracy and reducing pointing times. Practitioners may want to consider designing workstations, or portable computers, that require infrequent typing with a touchscreen interface. Portable computers could use the touchscreen for rapid cursor positioning, selections, graphical entry, and when necessary, typing alpha-numeric values. When a keyboard is not needed, the additional space could be used to display data. Touchscreens are clearly capable of far more than they have been used for, and should be considered for more diverse applications. However, careful attention must be paid to the resolution and response time when choosing a touchscreen. The amount of light transmitted through the touchscreen surface and types of stylus that can be used must also be considered (Sears, Plaisant, \& Shneiderman, 1991).

Additional studies may investigate using smaller touchscreen keyboards, various keyboard layouts, keys shapes and sizes, or various types of feedback. There are many potential improvements to touchscreen keyboards that may increase data entry speeds, reduce errors, reduce screen consumption, and improve user satisfaction.

\section{Potential improvements to touchscreen keyboards}

This study provides an estimate of typing rates using touchscreen keyboards. There are many potential improvements to touchscreen keyboards to be explored. First, shift keys were not provided (several other unused keys were also left off the keyboard). Shift keys could be used by touching the shift key followed by the key to be typed. The feedback provided to users could also be improved. In the current study both visual and audible feedback were provided. However, providing different feedback for certain keys may prove useful. Having a different tone for the space and done (return) keys, indicating closure of a word or line, and a third tone for the backspace key, indicating the correction of an error, may prove useful. Due to the size of the space bar, the visual feedback was slightly slower than other keys. This could be improved, by highlighting a smaller section of the key, not highlighting the key at all, or just flashing the border of the key. Making a touch anywhere outside of the keyboard to enter a space is another possibility, allowing spaces to be entered rapidly without touching a specific key.

The touchscreen technology used in this study could be improved in several important ways. The 
major problem, which is common to many different technologies, was the inability of the touchscreen to identify multiple touches. This made it impossible for users to rest their fingers on the screen, or the 'home row', and overlapping touches could not be recognized, forcing a short delay between touches. Capacitive touchscreens have been reported to be the slowest technology, compounding this problem (Carroll Touch, 1989). A technology that responds to touches more rapidly, or that allows the identification of simultaneous touches, even as few as two, may increase data entry rates. The slow response rate is suspected of being a major limit for the typing rate. If a different, faster responding, technology were used, it is believed that typing rates for the touchscreen could increase significantly.

\section{Acknowledgements}

I would like to thank the IwC reviewers, Linda Weldon and Catherine Plaisant for their comments, Ben Shneiderman for his guidance, and the subjects for their participation and patience. I would also like to thank NCR Corporation and the Museum of Jewish Heritage for partial support of this research.

\section{References}

Ahlström, B. and Lenman, S. (1987). Touch screen, cursor keys, and mouse interaction. Work with Display Units, Elsevier, New York, 831-837.

Ahlström, B. and Lenman, S. (1990). Fatigue when using a touch screen. Work in progress.

Battenberg, J. (1989). Touch screen versus keyboard: a comparison of task performance of young children. Journal of Special Education Technology, X(1), 24-28.

Beringer, D. and Peterson, J. (1985). Underlying behavioral parameters of the operation of touch-input devices: biases, models, and feedback. Human Factors, 27(4), 445-458.

Beringer, D., (1989). Touch panel sampling strategies and keypad performance comparisons. Proceedings of the 33rd Annual Meeting of the Human Factors Society. Santa Monica, CA, 71-75.

Beringer, D. \& Bowman, M. (1989). Operator behavioral biases using high-resolution touch input devices. Proceedings of the 33rd Annual Meeting of the Human Factors Society. Santa Monica, CA, 320322.

Card, S., Moran, T., \& Newell, A. (1983). The Psychology of Human-Computer Interaction. Hillsdale, NJ: Lawrence Erlbaum Associates.

Carroll Touch, (1989). Touch Handbook. Round Rock, TX: Carroll Touch Inc. 
Fitts, P. M. (1954). The information capacity of the human motor system in controlling the amplitude of movement. Journal of Experimental Psychology, 70, 193-242.

Gould, J., Greene, S., Boies, S., Meluson, A., and Rasamny, M. (1990). Using a touchscreen for simple tasks. Interacting with computers, 2(1), 59-74.

Grant, A., (1987). The computer user syndrome. Journal of the American Optometric Association, 58(11), 892-901.

Hall, A., Cunningham, J., Roache, R., and Cox, J. (1988). Factors affecting performance using touchentry systems: tactal recognition fields and system accuracy. Journal of Applied Psychology, 73(4), 711720.

Karat, J., McDonald, J., and Anderson, M. (1986). A comparison of selection techniques: touch panel, mouse, keyboard. International Journal of Man-Machine Studies 1, 73-92.

Muratore, D.A. (1987). Human performance aspects of cursor control devices, Mitre Corporation working paper 6321. Houston, TX.

Ostroff, D. \& Shneiderman, B. (1988). Selection devices for user of an electronic encyclopedia: an empirical comparison of four possibilities. Information Processing \& Management, 24(6), 665-680.

Revis, D., Swatski, J., Crittenden, R., Sears, A., \& Shneiderman, B. (1991). A Study of Touchscreen Typing using Different Size Keyboards. Work in Progress.

Sears, A., Shneiderman, B. (1991). High-precision touchscreens: Design strategies and comparisons with a mouse. International Journal of Man-Machine Studies, 34, 4.

Sears, A., Plaisant, C., \& Shneiderman, B. (1991). A new era for touchscreen applications: High precision, dragging icons, and refined feedback. To appear in: R. Hartson \& D. Hix (Eds.), Advances in Human-Computer Interaction, Vol. 3.

Tinker, M. (1956). Effect of sloped text upon the readability of print. American Journal of Optometry and Archives of the American Academy of Optometry, 189-195.

Weisner, S. (1988). A touch-only interface for a medical monitor. Proceedings of the Human Factors Society -- 32nd Annual Meeting. Santa Monica, CA, 435-439. 
